\title{
Effect of consuming high-fat diet on the morphological parameters of adrenal gland
}

\author{
Topal $\mathrm{F}^{1}$, Goren $\mathrm{H}^{2}$, Yucel $\mathrm{F}^{3}$, Sahinturk $\mathrm{V}^{4}$, Aydar $\mathrm{Y}^{3}$ \\ Department of Medical Services and Techniques, Vocational School of Health Services, Bilecik Seyh Edebali \\ University, Gülümbe, Bilecik, Turkey. fatma.topal@bilecik.edu.tr
}

\section{ABSTRACT}

OBJECTIVES: The incidence of obesity and obesity-assosiated pathologies continues to increase with profound adverse effects on health status in the developed countries.

BACKGROUND: We aimed to investigate the effect of high fat diet on the adrenal gland morphology.

METHODS: We fed the mice with either high-fat diet ( $60 \% \mathrm{kcal}$ from fat) or low-fat diet (10\% kcal from fat) for nine weeks. Unbiased stereological methods were used to evaluate the adrenal gland morphology. The sections were evaluated using Cavalieri's method and volume fraction approach. We calculated mean volume of

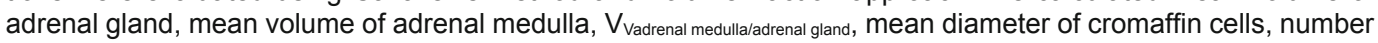
of chromaffin cells in per unit volume $\left(\mathrm{N}_{\mathrm{Vcc}} \mathrm{mm}^{-3}\right)$, total number of cromaffin cells, $\mathrm{V}_{\mathrm{V}_{z} \text { ona glomerulosa/adrenal cortex, }} \mathrm{V}_{\mathrm{V}_{z} \text { ona }}$ fasciculata/adrenal cortex, $\mathrm{V}_{\mathrm{V} z \text { ona reticulosa/adrenal cortex. }}$

RESULTS: The weight of adrenal gland, body weight intraperitoneal adipose tissue and adrenal gland weight in the obese mice significantly increased when compared with the control group. No changes were observed in the

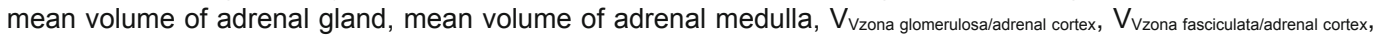
total number of cromaffin cells and diameter of cromaffin cells. However, $\mathrm{N}_{\mathrm{Vcc}_{\mathrm{cc}}} \mathrm{mm}^{-3}$ and $\mathrm{V}_{\mathrm{Vzona}}$ reticulosa/adrenal cortex in the obese mice considerably increased compared with the control group.

CONCLUSION: The present results suggest that high fat diet adversely affects the adrenal gland morphology (Tab. 2, Fig. 6, Ref. 28). Text in PDF www.elis.sk.

KEY WORDS: high-fat diet, morphology, obesity, adrenal gland, stereology.

\section{Introduction}

The prevalence of diseases associated with content of diet such as obesity, diabetes and cardiovascular diseases has increased significantly in recent years. The consumption of fast food with high fat content has made high-fat eating habits inevitable. Consumption of high-fat diet triggers obesity in adults. At the same time, high fat diet has been used in rodents for years to develop experimental obesity, dyslipidemia and insulin resistance (1-3). Obesity is shown to adversely affect over $35 \%$ of adult health in developed countries. Consumption of high-fat diet and obesity are known to be a major risk factor for the development of various forms of cancers, sleep disorders, non-alcoholic steatohepatitis,

${ }^{1}$ Department of Medical Services and Techniques, Vocational School of Health Services, Bilecik Seyh Edebali University, Gülümbe, Bilecik, Turkey, ${ }^{2}$ Department of Anatomy, Faculty of Medicine, Duzce University, Duzce, Turkey, ${ }^{3}$ Department of Anatomy, Medical School of Eskişehir Osmangazi University, Eskişehir, Turkey, and ${ }^{4}$ Department of Histology, Medical School of Eskişehir Osmangazi University, Eskişehir, Turkey

Address for correspondence: Y. Aydar, Department of Anatomy, Medical School of Eskişehir Osmangazi University, 26480 Eskişehir, Turkey. Phone: +90 5366504433

Acknowledgement: The present study was also financially supported by Eskisehir Osmangazi University Research Projects Center with the project code 2014-586. liver failure, cardiovascular diseases, reproductive disorders, genital diseases and type 2 diabetes. Therefore, ingestion of high-fat diet and development of diet-associated obesity are responsible for the global endangering of public health (2-5).

Adrenal glands consist of cortex and medulla with important functions in neuroendocrine modulation and they participate in the formation of the hypothalamic-pituitary-adrenal axis (HPA axis). The adrenal cortex consists of three layers from outside to inside, namely zona glomerulosa, which produces mineralocorticoids that help in the regulation of blood pressure and electrolyte balance, zona fasciculata, which synthesizes glucocorticoids, whose functions include the regulation of metabolism and immune system suppression, and zona reticularis, which is the innermost layer producing androgens that are converted to fully functional sex hormones in the gonads and other target organs. On the other hand, the adrenal medulla consists of chromaffin cells that function as the main source of the catecholamines, noradrenaline (norepinephrine) and adrenaline (epinephrine) (6-9).

Consumption of high-fat diet is shown to trigger the development of obesity through prompting the activation of the HPA axis and production of several hormones from the hypothalamus, pituitary and adrenal glands. Animal studies in rodents have indicated that intake of high-fat diet increases activity of the HPA axis through stimulating both basal and stress-induced HPA activities. High-fat diet is discovered to affect the activity of the 
HPA axis via inducing glucocorticoids synthesis. Moreover, the high-fat diet is also shown to cause chronic stress-related effects in the body; that is to say, consumption of high-fat diet triggers neuroendocrine metabolic changes similar to those observed in exposure to stress $(2,3,10-12)$

Glucocorticoids released from adrenal cortex are important modulators of energy balance in the body. They are shown to play a central role in energy metabolism through affecting the neural pathways involved in food intake and regulation of energy consumption. In addition, consumption of high-fat diet is revealed to increase secretion of adrenal glucocorticoids. Likewise, feeding rats with sucrose and high-fat diet for 12 days is stated to affect triglyceride metabolism via increasing lipid flow $(2,10,12,13)$.

Furthermore, obesity is associated with changes in plasma cortisol and aldosterone levels. Cortisol and other glucocorticoids induce the differentiation of pre-adipocytes into adipocytes, thereby inducing obesity through contributing to the increase in body mass. Not only are obese people shown to have increased levels of aldosterone, they also have an elevated rate of glucocorticoid production. There is also an increase in plasma catecholamine levels in obesity. Similarly, corticosterone and aldosterone levels in obese animal models have been shown to be elevated significantly $(5,14,15)$. Adrenalectomy is shown to reduce the secretion of corticosterone synthesized from the adrenal cortex. Amount of adipose tissue, lipid intake and body weight are disclosed to be decreased in experimental animals after adrenalectomy in addition to the decrease in triglyceride, leptin and body weight levels. These observations highlight the role of adrenal gland in lipid metabolism $(4,9)$.

There are restricted numbers of studies available regarding the effect of high-fat diet on various morphological parameters of the adrenal gland; therefore, in the present study, we aimed to study the consequence of consuming high-fat diet on various morphological parameters of the adrenal gland, including total volume, ratio of cortex volume to medullary volume, volume of the layers of the adrenal cortex in addition to number and mean diameter of the chromaffin cells in adrenal medulla.

\section{Materials and methods}

\section{Animals and groups}

The animals were obtained from the Medical and Experimental Research Center of Eskişehir Osmangazi University, Eskişehir, Turkey. For the present study, 16 unmated female Swiss albino strains (8-10 weeks old, weighing 22-25 g) were used. The mice were randomly assigned to the control group, which contained 8 mice fed with standard chow (Altromin, C 1090-10, $10 \%$ fat, Germany, $\mathrm{n}=8$ ) and high-fat diet (HFD) group, which also contained 8 mice but fed with high-fat diet (Altromin, C 1090-60, 60 $\%$ fat, Germany, $\mathrm{n}=8$ ) (Fig. 1 and 2). The chow diets were kept at $-21{ }^{\circ} \mathrm{C}$ during the experiment and the animals were allowed to feed on the diet based on determined maximal amount of daily need. While the animals in HFD group received $5.24 \mathrm{kcal}$ from per gram of high fat diet, in the control group, they received 3.52 kcal from per gram of the standard chow. Animal cages were main-

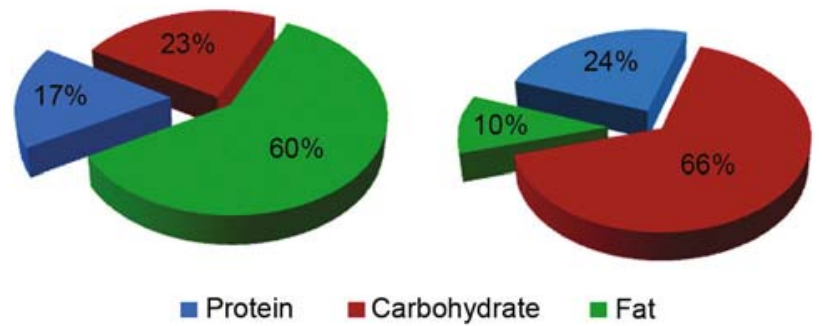

Fig. 1. Contents of the standard and high-fat diets
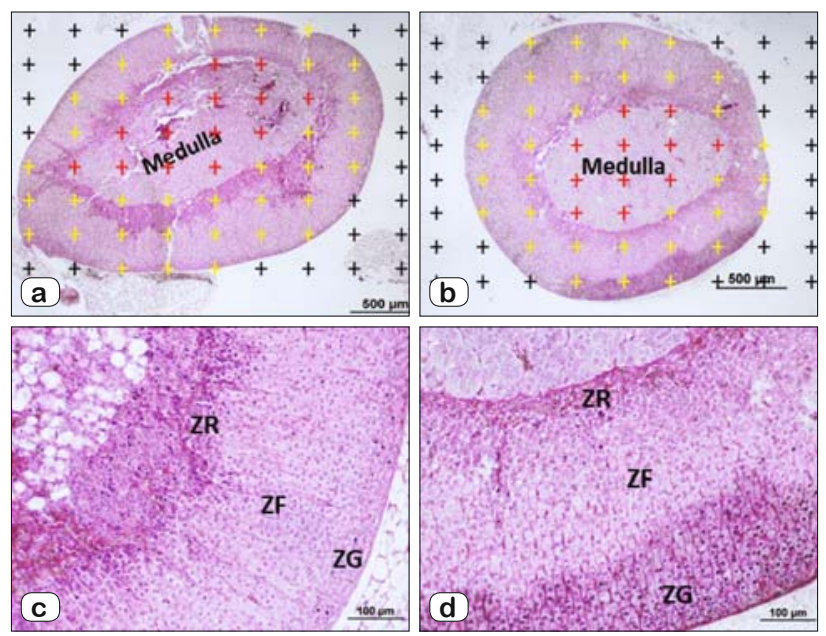

Fig. 2. Establishment of the mean volume of the adrenal gland using point counting method $(a, b)$, mean volume of the adrenal medulla $(a, b)$, ratio of the adrenal gland to adrenal medulla $\left(V_{v}\right)(a, b)$, ratio of the zona glomerulosa to adrenal cortex (c, d), ratio of the zona fasciculata to adrenal cortex (c, d), and ratio of the zona reticularis to adrenal cortex $\left(V_{v}\right)(c, d)$. Panel a shows $4 X$ magnification of a representative shot from the control group, Crossman triple stain, Bar: $500 \mu \mathrm{m}$, Panel b depicts $\mathrm{x} 4$ magnification of a representative shot from the experimental group, Crossman triple stain, Bar: $500 \mu \mathrm{m}$, Panel c illustrates 20X magnification of a representative shot from the experimental group, Crossman triple stain, Bar: $100 \mu \mathrm{m}$, finally Panel d displays $\times 20$ magnification of a representative shot from the experimental group, Crossman triple stain, Bar: $100 \mu \mathrm{m}$. ZG: zona glomerulosa, ZF: zona fasciculata, ZR: zona reticularis.

tained at $22 \pm 2{ }^{\circ} \mathrm{C}$ with a 12-hour light/12-hour dark cycle and the mice were fed with standard or high fat diet starting from the first day of the experiment while water was provided ad libitum (16). The treatment of the animals and experimental procedures were approved by the Experimental Animals Ethic Committee of Eskişehir Osmangazi University with the decision numbered 4011. The present study was also financially supported by Eskişehir Osmangazi University Research Projects Center with the project code 2014-586. While the care, feeding, and other procedures of the animals were carried out at the Experimental Animal Facility of the Medical and Experimental Research Center of Eskişehir Osmangazi University, the collection and stereological evaluation of the tissues in addition to the histological procedures were performed at the Anatomy and Histology Departments of the Medical School of Eskişehir Osmangazi University. 


\section{Histological procedures}

For the histological studies, the mice were anesthetized with an intraperitoneal injection of anesthetic cocktail prepared with $0.04 \mathrm{ml}$ xylazine and $0.2 \mathrm{ml}$ ketamine at the end of the 9-week feeding period. The adrenal glands were removed under anesthesia and then the mice were sacrificed with cervical dislocation. After removing the adrenal glands, they were carefully weighed with sensitive digital balance (Mettler Toledo). Consequently, the adrenal glands were fixed in $4 \%$ paraformaldehyde at $4{ }^{\circ} \mathrm{C}$ for 24 hours, dehydrated in alcohol, cleaned in xylene and then embedded in paraffin. All paraffin blocks were subjected to the fixation and processing steps under the same conditions. Subsequently, 5 - $\mu \mathrm{m}$ thick cross sections were cut off from the blocks based on the method of systematic random sampling and the sections were place on the poly-l-lysine-coated slides. The sections were stained with Crossman triple staining (17).

\section{Morphological analyses}

Adrenal sections were examined using Nikon ECLIPSE E400 microscope with drawing tube attachment at the Department of the Anatomy, Medical School of Eskişehir Osmangazi University. Before starting the analyses, a preliminary study was carried out to determine a suitable strategy for the current study. Accordingly, we decided to use Cavalieri principle to perform volume calculation. For this purpose, a 5-mm thick sections were obtained from the adrenal glands through the systemic random sampling method and a point-counting test grid was used. Average volume of the adrenal cortex and medulla was determined using the formula “A $=\Sigma \mathrm{P} \mathrm{x} \mathrm{d}{ }^{2}, \mathrm{~V}=\mathrm{T} \times \Sigma \mathrm{A}$ ” (Fig. 2) $(18,19)$.

Furthermore, $7 \times 7$ - point dot scale put on the sections was used to calculate Vv. To determine Vvmedulla/adrenal gland, the number of dots per adrenal medulla was divided by the number of dots falling into the adrenal gland. Likewise, $\mathrm{V}_{\text {Vzona glomerulosa }}$ was calculated by dividing the number of dots per zona glomerulosa to the dots falling into the adrenal cortex, $\mathrm{V}_{\text {Vzonafasciculata }}$ was determined by dividing the number of dots per zona fasciculata to the dots falling into the adrenal cortex, and to calculate $\mathrm{V}_{\mathrm{v}}$ the number of dots per zona reticularis was divided by the number of dots falling into the adrenal cortex (Fig. 2). These calculations were performed by taking the average of the total values obtained from each section in each animal as reported earlier $(20,21)$.

The mean diameters of the chromaffin cells were plotted using an objective counting frame at the draw setting of the microscope on 100X objective. We determined the major diameter (a) as the diameter passing through the long axis of the chromaffin cells and as the minor diameter (b) passing through midpoint of the major diameter and the short axis of the chromaffin cells. Afterwards, the mean diameter of the chromaffin cells was calculated using the major diameter (a) and the minor diameter (b) in formula $\mathrm{D}^{-}=\sqrt{a x b}$ as reported previosly (Fig. 3) (20, 21).

We used the formula $\mathrm{N}_{\mathrm{a}} / \mathrm{D}^{-}+\mathrm{t}$ to calculate the number of chromaffin cells per unit of volume. In the formula, $\mathrm{Na}$ is the number of cells per unit of volume, $\mathrm{D}^{-}$is the average nuclear diameter, and $t$ is the section thickness. We established the number of chromaffin cells falling into the unit area of various regions determined

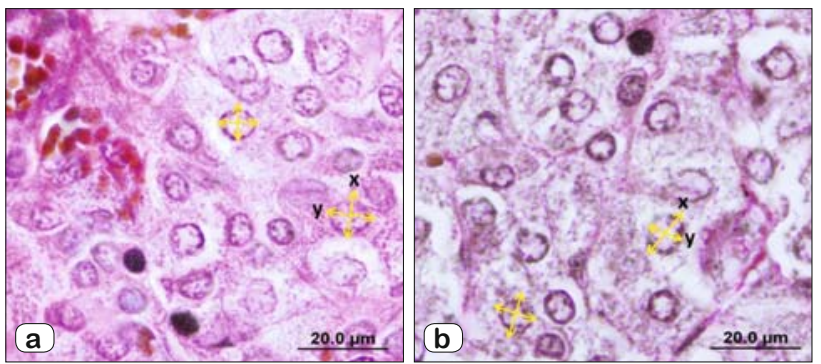

Fig. 3. Calculation of nuclear diameter of chromaffin cells and measurement of major $(x)$ and minor $(y)$ diameters $(a, b)$ : a: the standard control group, b: high fat-diet group at $\mathbf{x 1 0 0}$ magnification, Crossman triple stain, Bar: $20 \mu \mathrm{m}$
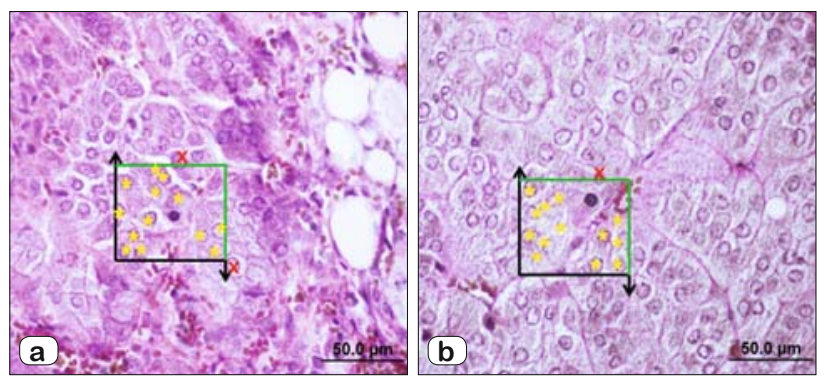

Fig. 4. Calculation of chromaffin cell number per unit area in standard control group (a) and high fat diet group (b): displays the number of chromaffin cells falling into the neutral count frame, $x$ shows the number of chromaffin cells excluded from the counting at $x 40$ magnification, Crossman triple stain, Bar: $20 \mu \mathrm{m}$

through the systematic random sampling method on the sections using 100X objective in the draw setting of microscope to estimate the number of chromaffin cells per unit of volume of the sections. While the chromaffin cells falling on the right and upper lines of the counting frame were counted, the cells falling on the left and bottom lines were not included in the count $(20,18)$. The same counting approach was applied to the sections obtained from each animal. Subsequently, we determined the number of chromaffin cells $\left(\mathrm{N}_{\mathrm{a}}\right)$ per unit area after establishing the number of chromaffin cells falling into the area of neutral counting frame by considering the magnification factor. The number of chromaffin cells per unit of volume $\left(\mathrm{N}_{\text {Vcromaffin cells }}\right.$ in $\left.1 \mathrm{~mm}^{-3}\right)$ was determined via placing the obtained data in the formula. Eventually, total chromaffin cell count was calculated by multiplying the number of cells per unit of volume and adrenal medulla volume (Fig. 4) (20,21).

\section{Ethics statement}

The animals used in the present study were handled and treated in accordance with the recommendations in the Guide for the Care and Use of Experimental Animals Ethic Committee of Eskişehir Osmangazi University (EAEC-ESOGU). The protocols used were approved by the Committee on the Ethics of Animal Experiments of Medical and Experimental Research Center of ESOGU. Institutional Ethical Committee number under which this study has been approved was 401-1. The mice were sacrificed by cervical dislocation under anesthesia with minimum suffering. 


\section{$593-600$}

\section{Statistical analyses}

The statistical analyses were carried out using IBM SPSS (Statistical Package for Social Sciences) program 21.Initially, Shapiro-Wilk normality test was applied to the groups to determine whether the data pertaining to the groups showed or did not show normal distribution. Since the data showed normal distribution, the data were analyzed using independent t-test. The results were considered within $95 \%$ confidence bounds and a $\mathrm{p}<0.05$ was considered to be statistically significant. The results were expressed as mean $\pm \mathrm{SEM}$.

\section{Results}

\section{The effect of high-fat diet on general parameters}

We recorded body weights $(\mathrm{BW})$ of the animals in control group (standard diet) and experimental (high-fat) group prior to and after the feeding period. Mean BW of the adult female mice was $28.62 \pm 2.34 \mathrm{~g}$ at the beginning of 9-week feeding period. While the mean BW was $34.12 \pm 3.13 \mathrm{~g}$ in experimental group, it was $29 \pm 1.19 \mathrm{~g}$ in the control group (Fig. 5A, Tab. 1). At the end of the feeding regime, the mean $\mathrm{BW}$ of the animals in the experimental group was significantly increased in comparison to the mice in the control group $(\mathrm{p}=0.008)(\mathrm{p}<0.05)$. Moreover, we removed intraperitoneal adipose tissue and adrenal glands at the end of the experiment and weighed them. Likewise, the fat tissue was markedly increased in the experimental group compared to the control group $(p=0 ; p<0.001)$ (Fig. 5B, Tab. 1). Similarly, while the weight of the adrenal gland was $0.013 \pm$ $0.092 \mathrm{~g}$ in the control group, it was $0.09 \pm 0.179 \mathrm{~g}(\mathrm{p}=0.017)$ $(\mathrm{p}<0.05)$ (Fig. 5C, Tab. 1) in the experimental group. The increase in the weight of the adrenal gland was statistically significant in the experimental group compared to the control and

Tab. 1. Sum of statistical analysis results regarding comparison of body weights, intraperitoneal adipose tissues, adrenal gland weights, mean volume of adrenal glands, mean volume of adrenal medullas, ratio of adrenal medulla to adrenal gland in standard control and high-fat diet groups. Note that the differences among the groups were evaluated using Mann Whitney $U$ test and $T$ tests and " $n$ " shows the number of the animals used in each group $(* * *: p<0.001, * *: p<0.01)$.

\begin{tabular}{|c|c|c|c|c|}
\hline & $\operatorname{SCD}(n=7)$ & $\operatorname{HFD}(n=8)$ & $\mathrm{p}$ & Sig \\
\hline Body weight (g) & $29 \pm 1.19$ & $34.12 \pm 1.11$ & $\mathrm{p}=0.008$ & $* *$ \\
\hline Intraperitoneal adipose tissue $(\mathrm{g})$ & $0.37 \pm 0.03$ & $1.69 \pm 0.24$ & $\mathrm{p}<0.001$ & $* * *$ \\
\hline Adrenal gland weight $(\mathrm{g})$ & $0.013 \pm 0.092$ & $0.09 \pm 0.198$ & $\mathrm{p}=0.017$ & $* *$ \\
\hline Mean volume of adrenal gland $\left(\mathrm{mm}^{3}\right)$ & $2.13 \pm 0.36$ & $2.09 \pm 0.23$ & $\mathrm{p}=0.82$ & n.s. \\
\hline Mean volume of adrenal medulla $\left(\mathrm{mm}^{3}\right)$ & $0.44 \pm 0.16$ & $0.33 \pm 0.17$ & $\mathrm{p}=0.24$ & n.s. \\
\hline Adrenal medulla/Adrenal gland ratio $\left(\mathrm{V}_{\mathrm{V}}\right)$ & $20.58 \pm 5.95$ & $15.41 \pm 7.02$ & $\mathrm{p}=0.15$ & n.s \\
\hline
\end{tabular}

n.s. not significant

A

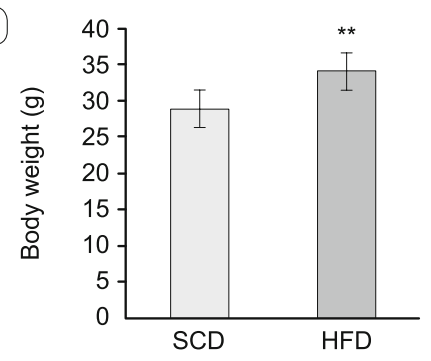

(D)

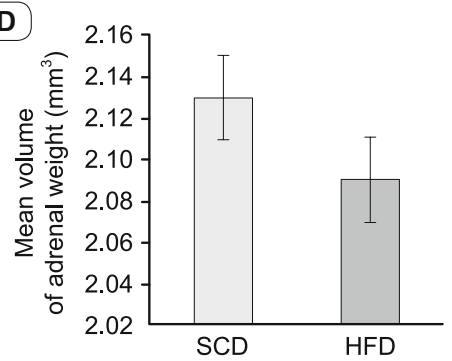

(B)

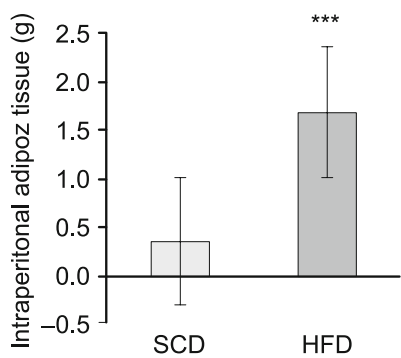

(E)

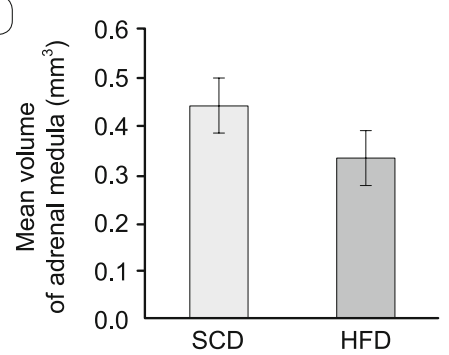

C

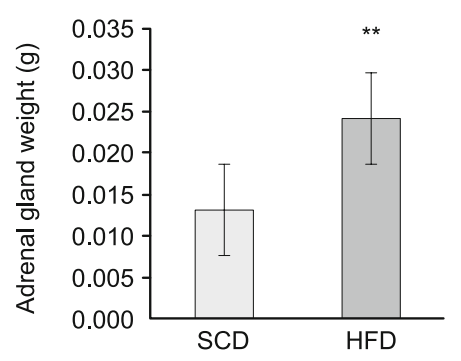

F

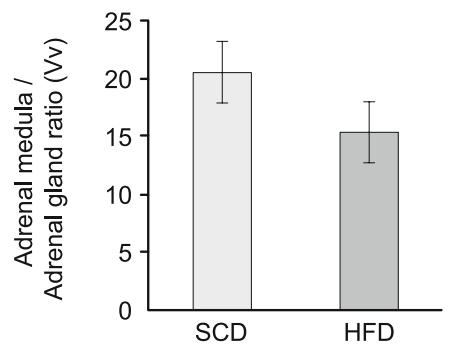

Fig. 5. The influence of high-fat diet on general parameters and morphology of adrenal gland: Notice that Panel A displays body weights (g) of the animals, Panel B shows weight (g) of intraperitoneal adipose tissue, Panel $\mathrm{C}$ depicts weight of the adrenal gland (g), Panel D demonstrates mean volume of adrenal gland $\left(\mathrm{mm}^{3}\right)$, Panel $E$ shows mean volume of adrenal medulla $\left(\mathrm{mm}^{3}\right)$, and Panel $F$ presents ratio of adrenal medulla to adrenal gland $\left(\mathrm{V}_{\mathrm{v}}\right)$. Values were expressed as mean \pm SEM and independent $\mathrm{T}$ test was applied for comparisons. SCD: standard control diet $(\mathrm{n}=7)$, HFD: high fat-diet $(\mathrm{n}=8),(* * * \mathrm{p}<0.001, * * \mathrm{p}<0.01)$. 
Tab. 2. Note that the table illustrates statistical analyses and their comparison concerning the nuclear diameter of chromaffin cells and their numbers per unit volume $\left(\mathrm{N}_{\mathrm{vcc}} \mathrm{mm}^{-3}\right)$, total chromaffin cell counts, ratio of zona glomerulosa to adrenal cortex, ratio of zona fasciculata to adrenal cortex, and ratio of zona reticularis to adrenal cortex. The differences between the groups were evaluated using independent $T$ test, $n$ : the number of animals used in each group $(*: p<0.05)$.

\begin{tabular}{lcccc}
\hline & $\mathrm{SCD}(\mathrm{n}=7)$ & $\mathrm{HFD}(\mathrm{n}=8)$ & $\mathrm{p}$ & Sig \\
\hline Nuclear diameter chromaffin cell $(\mu \mathrm{m})$ & $1.8 \pm 0.06$ & $1.84 \pm 0.1$ & $\mathrm{p}=0.81$ & $\mathrm{n} . \mathrm{s}$ \\
Numerical density of Chromaffin cell $\left(\mathrm{N}_{\mathrm{Vcc}} \mathrm{mm}^{-3}\right)$ & $12.76 \pm 2.64$ & $17.95 \pm 4.97$ & $\mathrm{p}=0.026$ & $*$ \\
Total chromaffin cell number & $5580.63 \pm 932.89$ & $5980.02 \pm 1464.89$ & $\mathrm{p}=0.99$ & $\mathrm{n} . \mathrm{s}$ \\
Glomerular zone/adrenal cortex Ratio $\left(\mathrm{V}_{\mathrm{v}}\right)$ & $21.88 \pm 4.66$ & $21.9 \pm 6.34$ & $\mathrm{p}=0.99$ & n.s \\
Fascicular zone/adrenal cortex Ratio $\left(\mathrm{V}_{\mathrm{v}}\right)$ & $57.07 \pm 7.08$ & $63.02 \pm 8.45$ & $\mathrm{p}=0.16$ & $\mathrm{n} . \mathrm{s}$ \\
Reticular zone/adrenal cortex Ratio $\left(\mathrm{V}_{\mathrm{v}}\right)$ & $21.03 \pm 5.19$ & $15.07 \pm 5.87$ & $\mathrm{p}<0.05$ & $*$ \\
\hline
\end{tabular}

n.s. not significant
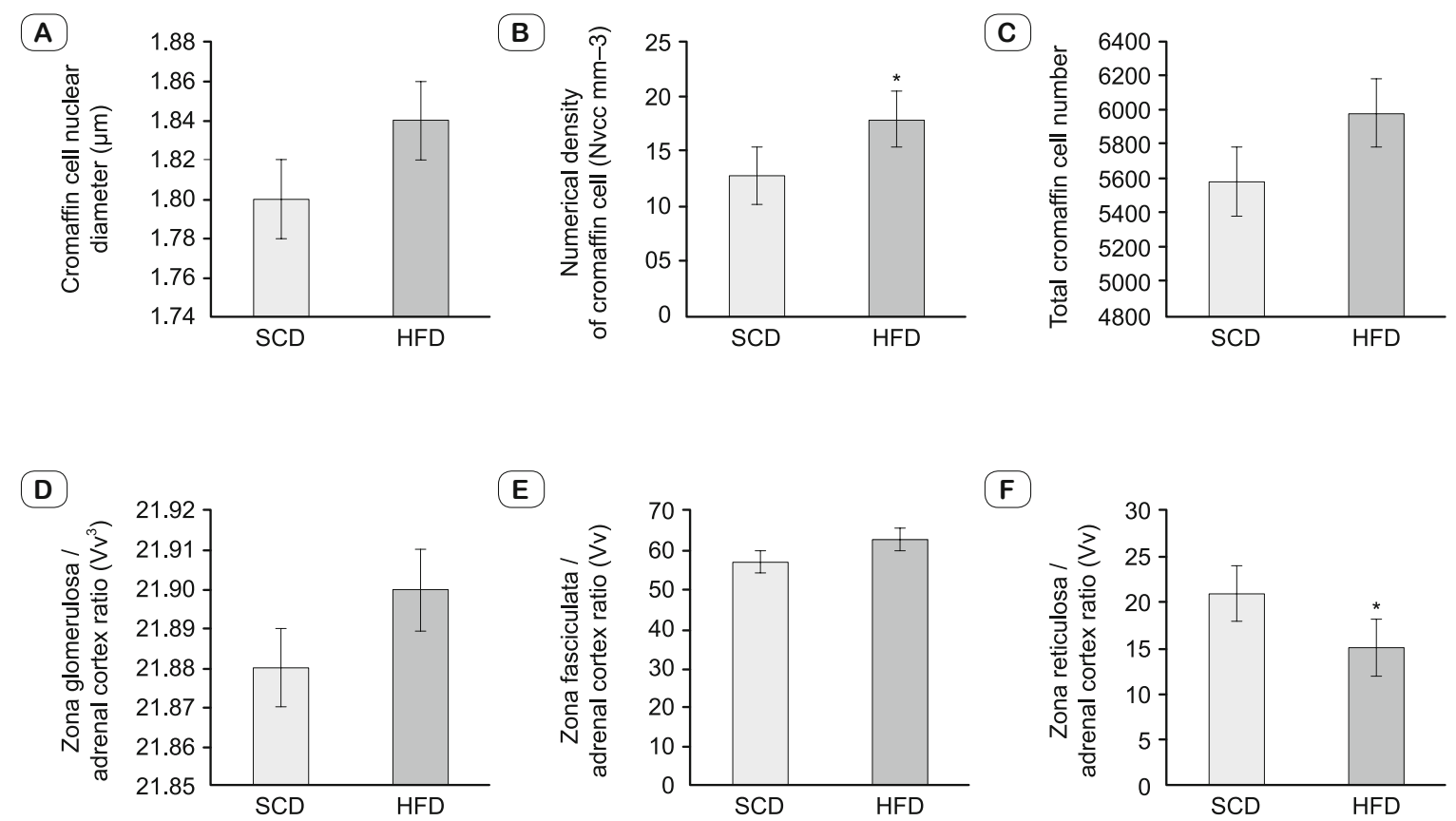

Fig. 6. The effect of high fat-diet on the morphology of chromaffin cells in adrenal cortex and adrenal medulla: Not that Panel A shows chromaffin Cell Nuclear Diameter $(\mu \mathrm{m})$, Panel B illustrates number of chromaffin cells per unit volume $\left(\mathbf{N}_{\mathrm{vcc}} \mathbf{m m}^{-3}\right)$, Panel $\mathbf{C}$ depicts total chromaffin cell number, Panel D displays ratio of zona glomerulosa to adrenal cortex, Panel E demonstrates ratio of zona fasciculata to adrenal cortex, and Panel $F$ shows ratio of zona reticularis to adrenal cortex. Values were expressed as mean $\pm \mathrm{SEM}$ and independent $\mathrm{T}$ test was applied for comparisons. SCD: standard control diet $(n=7)$, HFD: high fat-diet $(n=8),\left({ }^{*} p<0.05\right)$.

the increase was thought to result from the increase in body weight and fat tissue.

\section{Morphometric changes}

We used stereological methods to evaluate the effects of the high-fat diet exposure on the adrenal gland morphology. For this purpose, we first calculated the average volume of the adrenal glands of the animals. The mean volume of the adrenal glands in both groups was comparable. While the mean volume in the control group was $2.09 \pm 0.23 \mathrm{~mm}^{3}$, in the experimental group it was $2.13 \pm 0.36 \mathrm{~mm}^{3}$ (p>0.05) (Fig. 5D, Tab. 1). Even though there was an increase in the weight of the adrenal gland in the experimental group, it was not statistically significant.

Subsequently, we first calculated the volume of the adrenal medulla using the stereological analysis techniques and estab- lished that high-fat diet did not markedly affect the volume of the adrenal medulla. While the mean volume of the adrenal medulla was $0.33 \pm 0.17 \mathrm{~mm}^{3}$ in the experimental group, it was $0.44 \pm 0.16$ $\mathrm{mm}^{3}$ in the control group ( $\left.\mathrm{p}>0.05\right)$ (Fig. 5E, Tab. 1). On the other hand, we compared the ratio of the volume fraction of the adrenal medulla to adrenal gland to examine the effect of high-fat diet on the structural changes of the adrenal gland. The present analyses showed that intake of high-fat diet did not significantly affect the ratio of the adrenal medulla to adrenal gland, which was $20.58 \pm$ $5.95\left(\mathrm{~V}_{\mathrm{v}}\right)$ in the experimental group and $15.41 \pm 7.02\left(\mathrm{~V}_{\mathrm{v}}\right)$ in the control group $(\mathrm{p}>0.05)$ (Fig. 5F, Tab. 1). Overall, high-fat diet appeared to reduce the ratio of the adrenal medulla in the adrenal gland, but the decrease was not meaningful.

Furthermore, we performed stereological analyzes to investigate the effect of high-fat diet on chromaffin cells that reside in 
the adrenal medulla and synthesize catecholamines in the adrenal medulla. For this purpose; we calculated the nuclear diameter of the chromaffin cells, number of chromaffin cells per unit of volume, and the total number of chromaffin cells. While the nuclear diameter of chromaffin cells in the high-fat diet group was $1.84 \pm$ $0.1 \mathrm{~mm}$, it was $1.8 \pm 0.06 \mathrm{~mm}$ in the control group ( $\mathrm{p}>0.05$ ) (Fig. $6 \mathrm{~A}, \mathrm{Tab} .2)$. The current results showed that high-fat diet had no effect on the mean diameter of the chromaffin cells. By contrast, the number of the chromaffin cells per volume $\left(\mathrm{N}_{\mathrm{vcc}} \mathrm{mm}^{-3}\right)$ was considerably higher in the experimental group $(17.95 \pm 4.97)$ than in the control group $(12.76 \pm 2.64 ; \mathrm{p}=0.026 ; \mathrm{p}<0.05)$ (Fig. $6 \mathrm{~B}$, Tab. 2). We think that the increase in the number of the chromaffin cells per volume might correlate with the increase in fat level of the adrenal gland. Nevertheless, while the total number of chromaffin cells in the experimental group was $5980.02 \pm 1464.89$, it was $5580.63 \pm 932.89$ in the control group $(\mathrm{p}>0.05)$ (Fig. 6C, Tab. 2 ), demonstrating that high-fat diet did not affect the total number of chromaffin cells similar to their mean diameter.

We also stereologically examined morphology of the adrenal cortex known to generate various vital hormones highly important in the regulation of homeostasis in the body. For this purpose; we compared the ratio of zona glomerulosa to adrenal cortex, ratio of zona fasciculata to adrenal cortex and volume fraction of zona reticularis to adrenal cortex. Accordingly, the ratio of zona glomerulosa to adrenal cortex was $21.9 \pm 6.34$ in the experimental group and $21.88 \pm 4.66$ in the control group $(\mathrm{p}>0.05)$ (Fig. $6 \mathrm{D}$, Tab. 2), presenting no significant difference between the groups regarding the ratio of zona glomerulosa to adrenal cortex. Similarly, the ratio of zona fasciculata to adrenal cortex was comparable in the experimental group $(63.02 \pm 8.45)$ and the control group (57.07 $\pm 7.08 ; p>0.05)$ (Fig. 6E, Tab. 2). Conversely, while the ratio of zona reticularis to adrenal cortex was $15.07 \pm 2.07$ in the experimental group, it was $21.03 \pm 1.96$ in the control group $(\mathrm{p}$ $<0.05$ ) (Fig. 6F, Tab. 2), indicating that volume ratio of the zona reticularis to adrenal cortex was significantly decreased in the high-fat diet group. Whether the decrease in the relative volume of zona reticularis affects the generation of the androgens requires further studies.

\section{Discussion}

In the present study, we attempted to quantitatively analyze high-fat diet-associated morphological changes in the adrenal gland, which participates in significant neuroendocrine functions in the body. Current experimental setting obviously displayed that consumption of high-fat diet considerably increased not only body weight, adrenal gland weight but also intraabdominal fatty tissue. The increase in body weight and fatty tissue is shown to predispose the body to many diseases through inducing fat accumulation in intraabdominal organs, viscera and lead to obesity.

The present results showed that the intake of high-fat diet markedly increased body weight, intraabdominal adipose tissue and adrenal gland weight in adult female mice. The increase in body weight and intraabdominal fat tissue is shown to predispose the body to many diseases via causing obesity and visceral fat ac- cumulation. Moreover, the increase in weight of the adrenal gland, which is an important part of the HPA axis, might adversely affect the neuroendocrine functions $(4,5,10,12,22-26)$.

In the present study, we also calculated the volume of the adrenal gland, volume of its medulla, and rate of the adrenal medulla to the adrenal gland $\left(\mathrm{V}_{\mathrm{v}}\right)$ in the female rats fed with a high-fat diet for 9 weeks. Although there was an increase in the volume of the adrenal gland, its medulla, and rate of the adrenal medulla to the adrenal gland $\left(\mathrm{V}_{\mathrm{v}}\right)$, the increase was not statistically significant. There are several other studies consistent with our present observations. At their study examining the effect of high-fat diet intake on the size of adrenal gland, Swierczynska et al (2015) noted no significant change in the size of the adrenal medulla in the mice fed with high-fat diet for 18 weeks (5). Similarly, another recent study in rats indicated that consumption of high-fat diet for seven moths did not considerably change the thickness of adrenal medulla (23). On the other hand, at their study Diaz Aguila et al (2016) showed that feeding the rats with sucrose diet for 12 weeks increased collagen accumulation in the adrenal medulla, which thereby caused morphometric asymmetry; nevertheless, it did not affect the size of the adrenal medulla (27).

In the current study, we also calculated the average nuclear diameters of chromaffin cells, their number per unit of volume, and total number in the medulla of the adrenal glands in female mice fed with high-fat diet for 9 weeks. While we noted no meaningful difference in mean diameters of chromaffin cells and their total numbers, the number of chromaffin cells per unit of volume increased considerably in experimental group, indicating that high-fat diet increased level fat accumulation at organ level. Our literature review indicated no available study regarding diameters of chromaffin cells and their number per unit of volume of the adrenal medulla.

The chromaffin cells in the adrenal medulla synthesize catecholamines. In their study, Erdos et al show that plasma catecholamine levels are higher in obesity (14). This observation indicates morphological changes (change in total number of cells and increase in cell diameter) triggered in the chromaffin cell of the adrenal medulla in rats fed with high-fat diet. However, we observed no morphological changes in the adrenal medulla of rats fed with high-fat diet. This discrepancy might stem from variances in experimental settings, times, and dietary contents used in the current study. However, in their study Diaz Aguila et al (2016) detected no marked change in the cell density of adrenal medulla in the rats fed with a high-sucrose diet (27), a finding further supporting our observation that intake of high-fat diet did not considerable change the cell density of the chromaffin cells in the adrenal medulla.

In the present study, we performed stereological calculations to examine the effects of high-fat diet on the adrenal cortex layers. Accordingly, we calculated the rate of zona glomerulosa to adrenal cortex, zona fasciculata to the adrenal cortex, and zona reticularis to the adrenal cortex. The present results showed that while the rate of zona glomerulosa to adrenal cortex, and zona fasciculata to the adrenal cortex of the rats in experimental group were comparable with the controls, the rate of zona reticularis to 
the adrenal cortex was meaningfully reduced in the experimental group. Since zona reticularis is the layer producing androgens, a decrease in this layer might offer a quantitative explanation to the infertility and polycystic ovary triggered in obesity.

Besides, Swierczynskaet al. observed adrenal cortical hyperplasia, morphological changes in zona glomerulosa $(\mathrm{ZG})$ and zona fasciculata (ZF) of adrenal cortex in rats fed on a high-fat diet for 18 weeks. In the same study, while the authors noticed no significant change in the ZG area, they detected a considerable increase in expansion of the ZF area with no change in the cell density of the adrenal cortex (5). Likewise, Diaz Aguila et al (2016) observed no marked change in the thickness of ZG of adult rats fed on a high-sucrose diet for 12 weeks compared to control (27), an observation not consistent with our present findings. This discrepancy might stem from variances in experimental settings, times, and dietary contents used in the current study.

Besides, zona fasciculata constitutes the middle and largest zone of the adrenal cortex, residing directly beneath zona glomerulosa, and it mainly produces glucocorticoids (cortisol and corticosteroids), which regulates the metabolism of glucose, especially in the time of stress (e.g. part of the fight-or-flight response). In their study, Shin et al (2010) discover that the levels of serum corticosteroids are not changed in the rats fed with high-fat diet for 6 weeks (10). Likewise, Li et al (2016) report no significant change in blood cortisol levels of the rats fed with high-fat diet for 8 and 12 weeks (24). Adrenocorticotropic hormone (ACTH), released from the anterior pituitary upon stimulation induces secretion of glucocorticoids. Therefore, changes in the secretion of ACTH might also affect morphology of the adrenal cortex. However, Lomax et al (2013) noted no significant difference in the concentration of ACTH in pigs treated with high-fat diet for 12 weeks (28). On the other hand, Tannenbaum et al (1997) studied the effect of high-fat diet on the plasma levels of ACTH in rats fed with high-fat diet for 5 days, 1, 3, 9 or 12 weeks and noticed an increase in the level of ACTH (12). Morphological changes in different zones of the adrenal cortex might reflect alterations in the level of the hormones they generate. Accordingly, more or less preserved morphology in the adrenal glands of mice fed with high-fat diet in the present study is consistent with earlier studies showing no meaningful change in the levels of hormones generated in adrenal cortex $(10,24)$.

Aldosterone is secreted from the glomerulosa layer of the adrenal cortex. Clinical and experimental studies indicate that aldosterone levels are elevated in obesity, a finding that does not coincide with our present observation. This discrepancy might arise from differences in experimental settings, times, and dietary contents used in the present experiment and other experiments $(5,15)$.

Zona reticularis is the inner layer of the adrenal cortex and is shown to synthesize androgens. Therefore, the factors that interfere with neuroendocrine functions also affect zona reticularis of the adrenal gland. The high-fat diet is shown to trigger the development of obesity through the activation of HPA axis. Several studies indicate that consumption of high-fat diet leads to the development of chronic stress-like impacts and induces neuroendocrine metabolic changes through adversely affecting the HPA activity
$(2,12)$. These observations not only further support our current findings regarding the reduction in ratio of zona reticularis to the adrenal cortex but provide also a quantitative explanation at a physiological level.

Diaz Aguila et al (2016) revealed that feeding the rats with sucrose diet for 12 days increased the thickness of zona reticularis compared to the control group (27), an observation that is not consistent with our present observation. The reason for the conflict on thickness of zona reticularis might be due to the use of different diet for a discrete time.

In summary, in the present study we attempted to establish the effect of high-fat diet on various parameters of adrenal gland, its cortex, and medulla. The present study showed that high-fat diet increased body weight, amount of intraperitoneal fat tissue, and weight of the adrenal gland. By contrast, consuming high-fat diet did not seem to significantly alter the mean volume of the adrenal gland and adrenal medulla in addition ratio of the adrenal medulla to adrenal cortex. Likewise, mean diameter of the chromaffin cells and their number, ratio of zona glomerulosa and zona fasciculata to adrenal cortex was also comparable in experimental and control groups. However, the ratio of zona reticularis to adrenal cortex was significantly reduced in the experimental group. We believe the present study has contributed to the understanding of the effect of the high-fat diet on morphological parameters of the adrenal gland.

\section{References}

1. Ramalho L, da Jornada MN, Antunes LC, Hidalgo MP. Metabolic disturbances due to a high-fat diet in a non-insulin-resistant animal model. Nutr Diabetes 2017; 13 (3): e245.

2. Xu D, Xia LP, Shen L, Lei YY, Liu L, Zhang L, Magdalou J, Wang H. Prenatal nicotine exposure enhances the susceptibility to metabolic syndrome in adult offspring rats fed high-fat diet via alteration of HPA axis-associated neuroendocrine metabolic programming, Acta Pharmacol Sin 2013; 34 (12): 1526-1534.

3. Zhang L, Xu D, Zhang B, Liu Y, Chu F, Guo Y, Gong J, Zheng X, Chen L, Wang H. Prenatal food restriction induces a hypothalamic-pituitaryadrenocortical axis-associated neuroendocrine metabolic programmed alteration in adult offspring rats. Arch Med Res 2013; 44 (5): 335-345.

4. Yilmaz A, Suleyman H, Umudum Z, Sahin YN. The effect of adrenalectomy on leptin levels and some metabolic parameters in rats with diet-induced obesity. Biol Pharm Bull 2002; 25 (5): 580-583.

5. Swierczynska MM, Mateska I, Peitzsch M, Bornstein SR, Chavakis T, Eisenhofer G, Lamounier-Zepter V, Eaton S. Changes in morphology and function of adrenal cortex in mice fed a high-fat diet. Int J Obes (Lond) 2015; 39 (2): 321-330.

6. Dringenberg T, Schwitalla M, Haase M, Scherbaum WA, Willenberg HS. Control of CYP11B2/CYP11B1 expression ratio and consequences for the zonation of the adrenal cortex. Horm Metab Res 2013; 45 (2): 81-85.

7. Lalli E, Figueiredo BC. Pediatric adrenocortical tumors: what they can tell us on adrenal development and comparison with adult adrenal tumors. Front Endocrinol (Lausanne) 2015; 18 (6): 23.

8. Salman S, Buttigieg J, Nurse CA. Ontogeny of $\mathrm{O} 2$ and $\mathrm{CO} 2 / / \mathrm{H}+$ chemosensitivity in adrenal chromaffin cells: role of innervation. J Exp Biol 2014; 1; 217 (5): 673-681. 


\section{3-600}

9. Mitani F. Functional zonation of the rat adrenal cortex: the development and maintenance. Proc Jpn Acad Ser B PhysBiol Sci 2014; 90 (5): 163-183.

10. Shin AC, MohanKumar SM, Sirivelu MP, Claycombe KJ, Haywood JR, Fink GD, MohanKumar PS. Chronic exposure to a high-fat diet affects stress axis function differentially in diet-induced obese and diet-resistant rats. Int J Obes (Lond) 2010; 34 (7): 1218-1226.

11. Jankord R, Ganjam VK, Turk JR, Hamilton MT, Laughlin MH. Exercise training alters effect of high-fat feeding on the ACTH stress response in pigs. Appl Physiol Nutr Metab 2008; 33 (3): 461-469.

12. Tannenbaum BM, Brindley DN, Tannenbaum GS, Dallman MF, McArthur MD, Meaney MJ. High-fat feeding alters both basal and stressinduced hypothalamic-pituitary-adrenal activity in the rat. Am J Physiol 1997; 273 (6 Pt 1): E1168-1177.

13. Mantha L, Deshaies Y. Energy intake-independent modulation of triglyceride metabolism by glucocorticoids in the rat. Am J Physiol Regul Integr Comp Physiol 2000; 278 (6): R1424-1432.

14. Erdos B, Broxson CS, Cudykier I, Basgut B, Whidden M, Landa T, Scarpace PJ, Tümer N. Effect of high-fat diet feeding on hypothalamic redox signaling and central blood pressure regulation. Hypertens Res 2009; 32 (11): 983-988.

15. Goodfriend TL, Egan BM, Kelley DE. Aldosterone in obesity. Endocr Res 1998; 24 (3-4): 789-796.

16. Reeves PG, Nielsen FH, Fahey GC Jr. AIN-93 purified diets for laboratory rodents: final report of the American Institute of Nutrition ad hoc writing committee on the reformulation of the AIN-76A rodent diet. J Nutr 1993; 123 (11): 1939-1951.

17. Crossmon G. A modification of Mallory's connective tissue stain with a discussion of the principles involved. Anat Rec 1937; 69 (1): 33-38.

18. Mayhew T.M. The new stereological methods for interpreting functional morphology from slices of cells and organs. Exp Physiol 1991; 76 (5): 639-665.

19. Mandarim-de-Lacerda CA. Stereological tools in biomedical research. An Acad Bras Cienc 2003; 75 (4): 469-486.
20. Reid IM. Morphometric methods in veterinary pathology: a review. Vet Pathol 1980; 17 (5): 522-543.

21. Ulupinar E, Yucel F, Ortug G. The effects of prenatal stress on the Purkinje cell neurogenesis. Neurotoxicol Teratol 2006; 28 (1): 86-94.

22. la Fleur SE, Houshyar H, Roy M, Dallman MF. Choice of lard, but not total lard calories, damps adrenocorticotropin responses to restraint. Endocrinology 2005; 146 (5): 2193-2199.

23. Birem Z, Tabani K, Lahfa F, Djaziri R, Hadjbekkouche F, Koceir EA, Omari N. Effects of colocynth alkaloids and glycosides on Wistar rats fed high-fat diet. A biochemical and morphological study. Histochem Cytobiol 2017; 55 (2): 74-85.

24. Li S, Liao Y, Wang L, Huang R, Yue J, Xu H, Zhou H, Lou Z, Hu Y, Liu W. Dysregulation of Hypothalamo-Pituitary-Adrenocortical Axis in Overweight Female Diabetic Subjects is Associated with Downregulation of Corticosteroid Receptors and $11 \beta$-HSD1 in the Brain. Horm Metab Res 2016; 48 (3): 178-84.

25. Matias I, Petrosino S, Racioppi A, Capasso R, Izzo AA, Di Marzo $\mathbf{V}$. Dysregulation of peripheral endocannabinoid levels in hyperglycemia and obesity: Effect of high fat diets. Mol Cell Endocrinol 2008; 286 (Suppl 1-2): 66-78.

26. D'souza AM, Beaudry JL, Szigiato AA, Trumble SJ, Snook LA, Bonen A, Giacca A, Riddell MC. Consumption of a high-fat diet rapidly exacerbates the development of fatty liver disease that occurs with chronically elevated glucocorticoids. Am J Physiol Gastrointest Liver Physiol 2012; 302 (8): G850-863.

27. Díaz-Aguila Y, Castelán F, Cuevas E, Zambrano E, Martínez-Gómez M, Muñoz A, Rodríguez-Antolín J, Nicolás-Toledo L. Consumption of sucrose from infancy increases the visceral fat accumulation, concentration of triglycerides, insulin and leptin and generates abnormalities in the adrenal gland. Anat Sci Int 2016; 91 (2): 151-162.

28. Lomax MA, Karamanlidis G, Laws J, Cremers SG, Weinberg PD, Clarke L. Pigs fed saturated fat/cholesterol have a blunted hypothalamicpituitary-adrenal function, are insulin resistant and have decreased expression of IRS-1, PGC1 $\alpha$ and PPAR $\alpha$. J Nutr Biochem 2013; 24 (4): 656-663.

Received April 17, 2019. Accepted May 24, 2019. 